\title{
Nine Months of Memory Training Increases Cognistat Measured Memory in 79 - 89 Year-Old Mild Cognitively Impaired Individuals
}

\author{
Peter A. Magaro, Bruce Brotter, Mariyam Jalees \\ Memory Training Centers of America, New York, NY, USA \\ Email: petermagaro@memorytrainingcenters.com
}

Received 10 April 2015; accepted 5 May 2015; published 11 May 2015

Copyright (C) 2015 by authors and Scientific Research Publishing Inc.

This work is licensed under the Creative Commons Attribution International License (CC BY). http://creativecommons.org/licenses/by/4.0/

(c) (i) Open Access

\begin{abstract}
The effects of a memory training paradigm on performance across multiple cognitive domains, measured via the Cognistat, in 70 - 89 year-old individuals with mild cognitive impairment (MCI), were examined. Memory training sessions were conducted on average twice weekly, for 55 minutes each session, for 9 months. Across the testing period, Cognistat-measured memory increased relative to performance in other cognitive domains. Additionally, performance on nonmemory measures remained stable or declined. Thus, memory training in older adult, MCI individuals may result in improved memory, but not in improvement in other, non-memory, cognitive domains. Results replicate previous work examining "oldest-old" individuals ranging in age from 90 to 99 years old at the time of study start.
\end{abstract}

\section{Keywords}

Older Adult, Mild Cognitive Impairment, MCI, Cognistat, Cognitive Training, Memory

\section{Introduction}

Several studies have shown that memory training produces sizeable performance gains on memory tasks, as well as on cognitive tasks seemingly unrelated to memory [1]-[5]. It is not clear if these gains in performance across cognitive domains occur due to some aspects of the memory training techniques used previously, or whether these across-cognitive domain findings can be generalized to all memory training programs. Thus, it is not clear whether memory training increases performance on memory tasks specifically (specificity), or whether such training increases performance across multiple cognitive domains (generalization). This is an important question 
as the answer may have direct practical applications in clinical situations.

The research is equivocal regarding the answer for memory training and for training in other cognitive domains. For example, Smith et al. [6] examined the effects of training to improve speed of processing on attention and memory performance using the Repeatable Battery for the Assessment of the Neuropsychological Status (RBANS). It was concluded that speed of processing training affected all outcome measures, indicating that speed of processing training resulted in gains that generalized to the untrained measures of memory and attention, possibly because speed of processing was a component of these cognitive abilities. Additionally, beneficial effects of speed of processing training were the most evident at 3 months following training; interestingly, gains in memory were not evident at 3 months, suggesting that any cross-domain improvements following training may be temporary.

On the other hand, another set of studies reported that cognitive training improved task performance in areas specifically related to the cognitive domain of training, but did not improve performance in other domains. Specifically, Willis et al. [5] examined three training programs, with one each focusing on speed of processing, memory, and reasoning. It was found that, over five years, each training program increased performance only on its respective specified cognitive ability. Participants in all groups reported less difficulty performing everyday tasks. However, standard tests of function showed no differences in functional abilities between the groups. Furthermore, gains in memory performance in the memory group disappeared after ten years. Ball et al. [4] suggested that memory training was less effective than other types of cognitive training because the effects of training did not last for the same length of time as improvements did in other cognitive domains.

It was possible that the memory training used in the former studies was not particularly robust at increasing memory; thus, the beneficial effects of training on memory disappeared over time. In fact, other studies have demonstrated the effectiveness of memory training on memory performance. For example, Brehmer et al. [7] used a complex working memory task for 4 to 5 weeks as the memory training technique. Delayed and working memories were improved, as was attention. Unfortunately, although these results suggested that memory training might impact performance in other cognitive domains (i.e.: attention), direct comparisons between improvement in working memory and improvement on other cognitive tasks were not conducted [8]. Additionally, this previous work only examined older individuals without any cognitive deficits. The applicability of previous findings to individuals with mild cognitive impairment (MCI) is unclear; certainly however, the possibility that cognitive training can enhance cognition in cognitively impaired individuals is of clinical importance.

Thus, the current research addresses at least two questions: First, does cognitive training influence performance on tasks unrelated directly to the cognitive domain being trained? That is, does cognitive training generalize across cognitive domains? Second, how long do the effects of training last?

With regard to the former question, our prior work [9] indicated that, in individuals 90 years old and older with MCI, memory training resulted in increased memory performance on the Cognistast, but did not improve performance in other cognitive domains measured via the Cognistat. In that study, participants were trained biweekly using a computerized memory-training program. Approximately every three months, participants underwent cognitive testing. Results revealed increased memory performance as a function of time, but decreased or maintained performance on non-memory-related Cognistat measures. With regard to the latter question, in the same study [9], it was also reported that the effects of memory training were the most pronounced after 6 months of training with memory being particularly robust in these older individuals at this time. The present study examines these issues, using the same methods, in a population suffering from MCI, who are younger than those in former research, here, ages ranging from 79 to 89 years old.

Thus, the current study is an extension of prior work [9]. By examining a "younger" older population, we hope to generalize findings from our previous research to another age group. It is possible, however, that “older” versus "younger" populations will differ in their responses to a memory-training program. For example, the "oldest” old differ from the "younger" old in their pursuit of leisure activities [10], which may reflect their cognitive abilities, in addition to their overt physical abilities. On the other hand, Yang et al. [11] report that in two populations of older adults, both groups benefitted similarly from cognitive training. It should be noted that the populations in this latter study were considerably "younger", with the "oldest” group being about a decade younger than those individuals in our prior study. It is therefore not clear whether the memory training paradigm used here is able to modify memory in a group of individuals that is younger than that previously tested.

We hypothesized that, should memory training enhance cognition in this “younger” age group, it would do so by increasing memory performance, but not performance in other cognitive domains, replicating our previous findings [9]. We also anticipate similar effects regarding length of treatment and performance as those found 
previously.

\section{Methods}

\subsection{Participants}

Individuals receiving treatment in memory clinics established in senior living communities participated $(\mathrm{N}=68$, 48 women). Individuals were self-referred, or referred by either a family-member or senior living community staff member, for inclusion in the research. Inclusion criteria included age 70 to 89 years old at the start of testing $(\mathrm{M}=83.8, \mathrm{SD}=4.5)$, self-reported memory loss, a level of cognitive impairment that was determined through cognitive and neuropsychological testing to be mild-to-moderate, and completion of 3 cognitive testing times, post-initial evaluation, wherein all Cognistat Subscales were completed (with the exception of the Construction Subscale, see Analyses for additional information). This latter criterion was established to ensure a length of time sufficient for examining the effects of the memory training. Thus there were approximately 9 months intervening between initial testing (Time 1) and the last follow-up testing (Time 4; see Results for additional information regarding the length of time between testing sessions). Mild-to-moderate memory loss was used as inclusion criteria in order to maintain consistency regarding overall level of impairment at the start of treatment. Clinical and neuropsychological assessments were conducted to determine the level of cognitive impairment in the mild-to-moderate range, and to exclude diagnoses of age-related memory loss or dementia. Other exclusion criteria included intellectual deficiency or the presence of severe psychiatric disorder. All participants presented with normal or corrected visual and/or auditory acuity adequate to participate in the study, and no participants were taking any psychotropic medication known to impair cognition. See Table 1 for general demographical information of participants.

\subsection{Materials and Procedures}

Participants were interviewed by a licensed master's level mental health clinician in an initial evaluation session, during which consent forms were completed, medical history was gathered, and patients were asked to report and describe their presenting complaint. Upon referral for cognitive evaluation, the need for consult and consent from a Power of Attorney (POA) was determined by confirmation of this status with the Wellness Office of the community where the participant resided. If a POA was assigned, the POA was contacted to provide proper consent for participation. Participants were then referred to a doctoral level clinical psychologist for the administration of cognitive testing. Individuals presenting with memory loss in the diagnostic range of mild-moderate cognitive impairment, and having the inclusion criteria described above, were then referred for the memory training.

As part of the cognitive evaluation, all participants completed the Cognistat, which is a cognitive instrument normed by age that evaluates five domains of cognitive functioning, including language, construction, memory, calculation, and reasoning abilities, and with independent measures, assesses levels of consciousness, orientation, and attention. The 25-minute test produces a profile of cognitive abilities, rather than one global score [12] [13], and it is designed so that a patient's successful performance in the cognitive domains does not conceal deficits in others. The scoring system calculates values, ranging from 0 to 12, for each cognitive domain [14] [15].

Participants engaged in the development of a training plan with their clinician as the starting point of the memory training procedure. The training plan specified areas of concern and goals related to memory loss and focused on skills of daily living that were compromised by memory loss.

Training sessions took place on average twice weekly, for 55 minutes (See Results). Sessions were led by either doctoral level psychologists or master's level mental health clinicians. There are three components of memory training: computerized exercises, non-computerized training, and pragmatic strategies. The computerized component consists of a proprietary internet-based program of exercises that was developed to target the patient's capacity for storage of new information (delayed memory) and working memory. Nineteen exercises are designed to help with: verbal memory, visual memory, combined verbal and visual memory, auditory memory, attention and concentration. The exercises were constructed to have ten levels of difficulty, with consistent rules allowing participants to progress (or not) to the next level of difficulty.

The clinician monitored the participant's engagement in the process and, together, they selected exercises that provided an appropriate level of challenge for the participant. The clinician presented the stimuli of the exercises on the computer, and the participant responded with responses then entered into the computer by the clinician. 
Table 1. Demographic characteristics of study sample.

\begin{tabular}{|c|c|c|}
\hline Characteristic & $\mathrm{N}$ & $\%$ \\
\hline \multicolumn{3}{|l|}{ Age } \\
\hline $70-74$ & 2 & $3.3 \%$ \\
\hline $75-79$ & 9 & $14.8 \%$ \\
\hline $80-84$ & 19 & $31.1 \%$ \\
\hline $85-89$ & 31 & $50.8 \%$ \\
\hline \multicolumn{3}{|l|}{ Gender } \\
\hline Female & 48 & $78.7 \%$ \\
\hline Male & 13 & $21.3 \%$ \\
\hline \multicolumn{3}{|l|}{ Marital Status } \\
\hline Single & 2 & $3.3 \%$ \\
\hline Married & 8 & $13.1 \%$ \\
\hline Divorced & 4 & $6.6 \%$ \\
\hline Widowed & 31 & $50.8 \%$ \\
\hline Information not available & 16 & $26.2 \%$ \\
\hline \multicolumn{3}{|l|}{ Education } \\
\hline$<$ HS Level Education & 2 & $3.3 \%$ \\
\hline HS Grad & 26 & $42.6 \%$ \\
\hline Some College & 12 & $19.7 \%$ \\
\hline College Graduate & 21 & $34.4 \%$ \\
\hline \multicolumn{3}{|l|}{ Occupation (Previous) } \\
\hline Skilled/Unskilled worker & 7 & $11.5 \%$ \\
\hline Clerical & 6 & $9.8 \%$ \\
\hline Professional & 19 & $31.1 \%$ \\
\hline Other & 13 & $21.3 \%$ \\
\hline Information not available & 16 & $26.2 \%$ \\
\hline \multicolumn{3}{|l|}{ Physical Activity } \\
\hline Engages in regular exercise & 33 & $54.1 \%$ \\
\hline Does not engage in physical exercise & 11 & $18.0 \%$ \\
\hline Information not available & 17 & $27.9 \%$ \\
\hline \multicolumn{3}{|l|}{ Cognitive Activity } \\
\hline $\begin{array}{c}\text { Engages in cognitive stimulation such as reading, board games/ } \\
\text { word games }\end{array}$ & 33 & $54.1 \%$ \\
\hline Does not engage in cognitive stimulation & 13 & $21.3 \%$ \\
\hline Information not available & 15 & $24.6 \%$ \\
\hline \multicolumn{3}{|l|}{ Cardiovascular Condition } \\
\hline Yes & 10 & $16.4 \%$ \\
\hline No & 37 & $60.7 \%$ \\
\hline Information not available & 14 & $23.0 \%$ \\
\hline \multicolumn{3}{|l|}{ Medication to Treat Memory Loss } \\
\hline Yes & 9 & $14.8 \%$ \\
\hline No & 32 & $52.5 \%$ \\
\hline Information not available & 20 & $32.8 \%$ \\
\hline
\end{tabular}


Additionally, the clinician was able to regulate the degree of delay or distraction so that the participant experienced an optimal level of cognitive exercise for both working memory and delayed memory. An example of one of the 19 computer exercises was to present the participant with pictures of faces, with names, and then present the faces without names, challenging the participant to remember the correct face/name pairs. At level one there were 2 faces, with significant differences between the faces regarding gender, age and race. At higher exercise levels, the number of faces/names would increase, and the diversity among the faces would decrease.

In addition to the computer-generated exercises, clinicians provided non-computerized memory training, including 3 and 4-word retrieval exercises, inquiry regarding recent events (supported by cues to encourage the patient's ability to retrieve information), and other interactive memory-exercises based on the participant's treatment goals or area of interest. Training goals and areas of interest were determined by interaction with the participant. For example, a participant who was previously a managing partner in a law firm was provided with an intervention of the clinician reading passages of legal cases, and challenging the participant to recall the information from the passage.

Additional, practical strategies, for memory improvement were also presented. For example, participants were asked to identify a place in their apartment to keep important items (that were otherwise frequently misplaced), instructed to use a calendar, wear a watch, utilize appointment cards, and/or carry a schedule of the day's activities.

Cognitive testing was routinely re-administered, generally on a quarterly basis, though there was some variation in time intervals between testings, based on patient availability and other scheduling constraints (See Table 2). At the time of cognitive testing, the individual's training plan was also revised with the clinician to determine the individual's subjective experience of progress, while the testing report provided the objective measure of training effects.

In the current study, results from the Cognistat are examined. This test was administered during the initial assessment, in addition to being administered three additional times over the course of approximately 9 months (every three months). Thus, all participants completed the Cognistat 4 times; Time 1 being initial evaluation, and Times 2, 3, and 4 being those Times following memory training after approximately 3, 6, or 9 months, respectively.

\section{Analyses}

The Cognistat consists of several subscales, with those analyzed being Memory, Orientation, Attention, Communication, Repetition, Naming, Calculation, Similarity, and Judgment (Construction was not included in analyses because an additional 4 individuals did not complete all testing Times for this measure). Two additional individuals were eliminated for scoring "0" on subscales during the initial Time 1 testing, and one additional individual was eliminated because the memory scores were between four and five standard deviations above the mean, final $\mathrm{N}=61$.

Repeated-measures ANOVAs were performed on raw scores as a function of subscale, comparing performance across Time for each subscale. Additionally, correlations between subscale performance and number of training sessions per week were also performed. Raw score analyses do not allow comparisons between subscales, as subscales differ in their range (See Table 3 for Means and standard deviations of raw scores as a function of Cognistat Subscale and Time).

Therefore, improvement, relative to Time 1, at Times 2, 3, and 4, was calculated for each subscale. Such calculations allow standardization of subscales, enabling comparisons between subscales, and enables initial testing to act as a control condition for subsequent performance. Improvement was calculated via the formula (((Time $\mathrm{X}$ divided by Time 1) times 100) minus 100) (See Table 4 for Means and standard deviations as a function of Cognistat subscale and time for these improvement scores). A repeated measures ANOVA (27 levels formed by the combination of factors of subscale (9; Memory, Orientation, Attention, Communication, Repetition, Naming, Calculation, Similarity, and Judgment) and Time (3; improvement relative to Time 1 for Times 2, 3, and4)

Table 2. Means and standard deviations (SD) for average training sessions per week as a function of time.

\begin{tabular}{|c|c|c|c|c|c|}
\hline & Time 1 - 2 & Time 2 - 3 & Time 3 - 4 & Cumulative Time 1 - 3 & Cumulative Time 1 - 4 \\
\hline $\begin{array}{l}\text { Average Training } \\
\text { Sessions Per Week }\end{array}$ & $3.92(2.70)$ & $4.92(2.64)$ & 5.52 (3.33) & $4.15(2.02)$ & $4.47(2.01)$ \\
\hline
\end{tabular}


Table 3. Means and standard deviations (SD) of raw scores as a function of cognistat subscale and time.

\begin{tabular}{ccccc}
\hline & Time 1 & Time 2 & Time 3 & Time 4 \\
\hline Memory & $5.64(2.63)$ & $5.79(2.62)$ & $6.08(2.67)$ & $5.64(3.08)$ \\
Orientation & $9.48(3.10)$ & $9.33(3.32)$ & $8.70(3.68)$ & $8.43(3.68)$ \\
Attention & $7.48(1.41)$ & $7.34(1.26)$ & $7.49(1.10)$ & $7.33(1.27)$ \\
Communication & $5.46(0.96)$ & $5.54(0.90)$ & $5.70(0.76)$ & $5.43(1.24)$ \\
Repetition & $11.59(1.33)$ & $11.84(1.22)$ & $11.57(1.68)$ & $11.41(2.04)$ \\
Naming & $8.12(1.56)$ & $8.23(1.11)$ & $7.92(1.46)$ & $7.90(1.66)$ \\
Calculation & $3.46(1.31)$ & $3.43(1.19)$ & $3.13(1.19)$ & $3.03(1.28)$ \\
Similarity & $5.93(1.68)$ & $6.02(1.75)$ & $5.92(1.96)$ & $5.66(1.90)$ \\
Judgment & $4.97(1.15)$ & $5.13(.83)$ & $5.16(.95)$ & $4.93(1.26)$ \\
\hline
\end{tabular}

Table 4. Means and standard deviations (SD) of improvement scores as a function of cognistat subscale and time.

\begin{tabular}{cccc}
\hline & Time 2 & Time 3 & Time 4 \\
\hline Memory & $13.10(63.06)$ & $22.99(58.78)$ & $10.24(58.80)$ \\
Orientation & $1.74(32.36)$ & $-7.34(35.83)$ & $-7.17(46.79)$ \\
Attention & $3.26(35.67)$ & $4.93(35.18)$ & $1.10(25.42)$ \\
Communication & $4.86(28.62)$ & $8.69(30.09)$ & $2.38(30.30)$ \\
Repetition & $2.78(10.78)$ & $0.13(13.86)$ & $-1.71(14.76)$ \\
Naming & $7.46(44.70)$ & $3.42(44.80)$ & $-0.10(26.90)$ \\
Calculation & $5.76(41.10)$ & $-2.38(44.01)$ & $-6.74(41.36)$ \\
Similarity & $810(41.02)$ & $3.20(39.63)$ & $0.68(39.28)$ \\
Judgment & $13.91(65.15)$ & $18.25(84.91)$ & $8.25(59.60)$
\end{tabular}

was conducted. Comparisons between subscales at each Time were conducted via 3 (one for each Time) repeated measures ANOVAS (9: subscale). Repeated measures ANOVAs (Time: improvement relative to Time 1 for Times 2 vs. 3 vs. 4) were also conducted on each subscale.

Additionally, correlations were conducted between average number of training sessions per week and improvement scores as a function of Time and subscale as well. The average number of training sessions per week as a function of Time was calculated via the formula (number of training sessions between Times $\mathrm{X}$ and $\mathrm{Y}$ divided by number of weeks intervening between Times X and Y). Finally, correlations were also performed between age and education variables and memory improvement.

\subsection{Results}

There were no significant correlations between age and education, and any measure of memory.

\subsection{Raw Scores}

\subsubsection{Repeated ANOVAS}

For the Memory subscale, examination of means revealed increased memory as a function of Time; however, the repeated-measures ANOVA examining Memory performance was not significant $(p>0.05)$. The repeated measures ANOVA examining Orientation performance was significant $(\mathrm{F}(3,180)=4.90, \mathrm{p}<0.01)$, with examination of simple effects indicating significantly decreased Orientation as a function of Time (T4 $<\mathrm{T} 2, \mathrm{~T}(60)$ $=2.48, \mathrm{p}<0.05, \mathrm{~T} 3<\mathrm{T} 2, \mathrm{~T}(60)=2.55, \mathrm{p}<0.05)$. The ANOVA examining Calculation was also significant $(\mathrm{F}$ $(3,180)=4.09, \mathrm{p}<0.01)$. Examination of simple effects again indicated decreased performance as a function of Time $(\mathrm{T} 4<\mathrm{T} 2, \mathrm{~T}(60)=2.47, \mathrm{p}<0.05, \mathrm{~T} 3<\mathrm{T} 2, \mathrm{~T}(61)=2.46, \mathrm{p}<0.05)$. 


\subsubsection{Correlations}

The average number of training sessions per week between Times 3 and 4 was positively correlated with performance on the Attention subscale at Time $4(r=0.27, \mathrm{p}<0.05)$, and negatively with the Calculation subscale at Time $4(r=-0.27, \mathrm{p}<0.05)(\mathrm{N}=55$ due to loss of data of either number of training sessions or number of weeks intervening between test sessions).

\subsection{Improvement Scores}

\subsubsection{Repeated ANOVAS}

A repeated measures ANOVA encompassing all Times and all subscales was significant $(\mathrm{F}(26,1560)=1.86$, $\mathrm{p}$ $<0.01$ ). Planned comparisons between the Memory subscale at all Times and the other subscales revealed that i) Memory improvement at Time 2 was superior to improvement on Orientation at Time $3(t(60)=2.40, p=0.02)$; ii) Memory improvement at Time 3 was superior to improvement on seven of the other subscales at Time 4 (paired t-tests, p values ranging from 0.003 to 0.02 ), with the only non-significant subscale being Judgment (See Figure 1).

Improvement scores for each Cognistat Subscale were analyzed in three (Times 2, 3, and 4) one-way repeated measure ANOVAs in order to further clarify the subtests results. Planned comparisons between the Memory subscale and the other subscales at Time 2 revealed no significant differences. However, the ANOVA for Time 3 was significant $(\mathrm{F}(8,480)=2.66, \mathrm{p}<0.01$ (See Figure 2). Analyses of simple effects via a series of paired t-tests revealed Memory improvement was significantly greater than improvement on the subscales of Orientation $(t(60)=3.33, \mathrm{p}<0.01)$, Attention $(t(60)=2.29, \mathrm{p}=0.03)$, Naming $(t(60)=1.93, \mathrm{p}=0.058)$, Repetition $(t$ $(60)=3.11, \mathrm{p}<0.01)$, and Calculation $(\mathrm{t}(60)=2.50, \mathrm{p}=0.02)$. Although the ANOVA for Time 4 was not
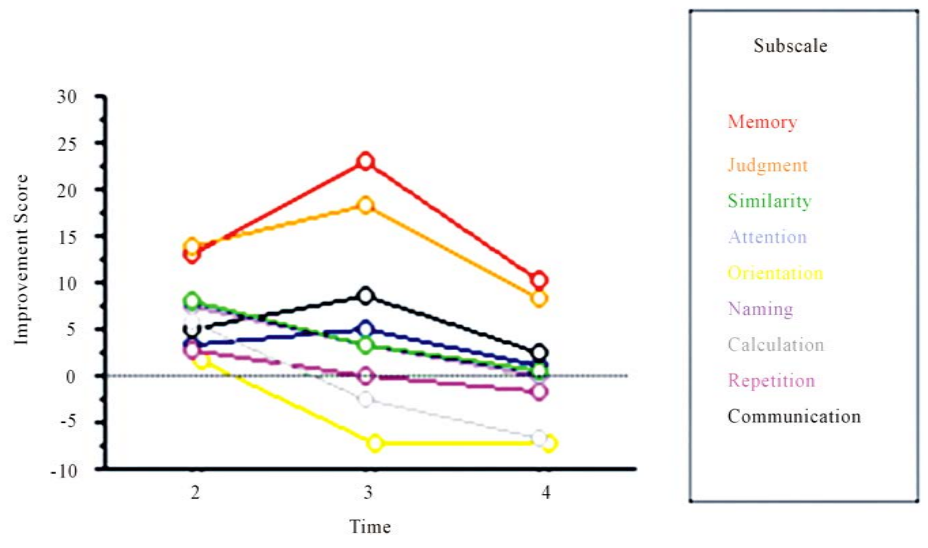

Figure 1. Improvement score as a function of time and cognitive subscale.

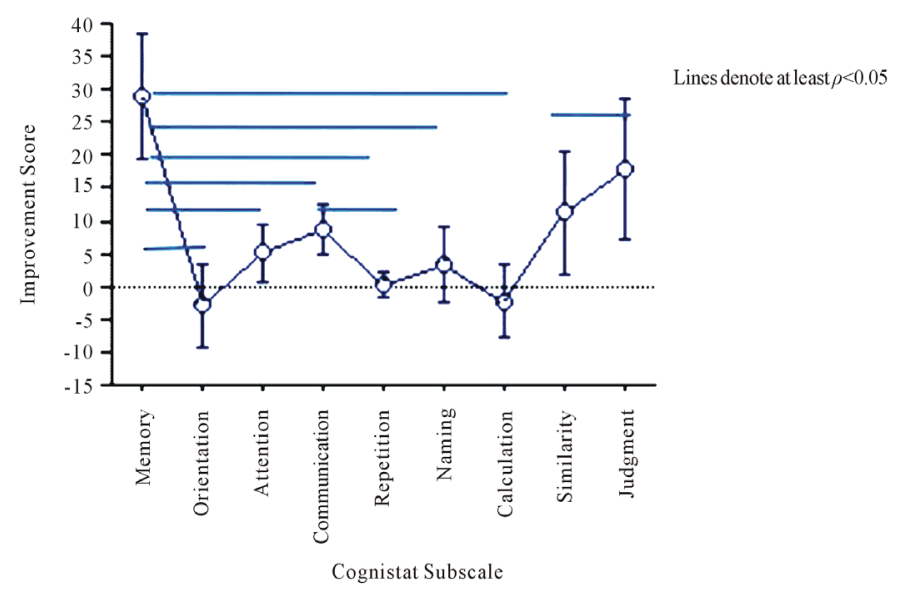

Figure 2. Time 3 Improvement score as a function of cognistat subscale. 
significant, planned comparisons between improvement on the Memory subscale and the other subscales at Time 4 revealed that Memory improvement at Time 4 was significantly greater than Orientation improvement $(t$ $(60)=2.16, \mathrm{p}<0.05)$.

Within-Subscale ANOVAs revealed Orientation improvement significantly decreased at Time 3, relative to Time $2(\mathrm{~F}(2,120)=3.01, \mathrm{p}=0.053 ; \mathrm{t}(60)=2.40)$. Calculation improvement also changed as a function of Time $(\mathrm{F}(2,120)=4.01, \mathrm{p}<0.05$, with performance decreasing at Times 3 and 4 compared with Time $2(t(60)=2.28$, $\mathrm{p}<0.05 ; t(60)=2.52, \mathrm{p}<0.05$, respectively). Repetition subscale improvement decreased as a function of Time $(\mathrm{F}(2,120)=3.33, \mathrm{p}<0.05$. Analyses of simple effects revealed that Time 4 Repetition improvement significantly decreased relative to Time 2 (paired t-test $(t(60)=2.48, \mathrm{p}<0.05$,). Similarly, Naming improvement also decreased as a function of Time $(\mathrm{F}(2,120)=2.95, \mathrm{p}=0.056)$. Time 3 Naming improvement decreased relative to Times 2 (paired-t test $(t(60)=2.79), \mathrm{p}<0.01)$, and Time 4 (paired t-test $(t(60)=2.10, \mathrm{p}<0.05$ ).

\subsubsection{Correlations}

Improvement in Communication at Time 2 was positively and significantly related to average number of training sessions per week between Times 1 and $2(r=0.29, \mathrm{p}<0.05)$. No other relationships were significant $(\mathrm{N}=$ 55 due to loss of data of either number of training sessions or number of weeks intervening between test sessions).

\section{Discussion}

The pattern of results indicates that the memory training procedure used here improves memory, but does not improve performance in other cognitive domains. Cognistat-measured memory performance increased, relative to the other Cognistat-measured cognitive domains, over 9 months. Replicating our prior work [6], this improved memory was the most evident at the 6th month testing. Specifically, improvement in memory 6 months after starting memory training was greater than improvement in five other cognitive domains. Although memory improvement at 9 months numerically decreased relative to memory improvement at 3 and 6 months, this decrease was not significant and, importantly, memory performance at 9 months remained significantly greater than orientation at the same time. Given the nature of MCI, the slight decrease in memory improvement at 9 months in these individuals could be a result of a ceiling effect [16]. Future work could address this possibility. Also of particular interest was the finding that memory improvement at 6 months was significantly greater than improvement on seven other cognitive domains at 9 months following training. In clear contrast to the increasing performance in the domain of memory, performance on four of the other subtests demonstrated significantly decreased performance over time.

These findings argue for the robust nature of the memory training procedure used here on memory performance specifically, relative to performance in other cognitive domains. Furthermore, these are important clinical findings and are deserving of replication using other cognitive testing methodologies. The additional implication is that not only is memory training specific to the domain of cognition, but that other cognitive functions can decline while a cognitive function undergoing training improves.

It was worth noting that judgment was the only cognitive domain that did not differ in improvement compared with memory. One potential reason for this finding may be that memory is critical for judgment decisions and, therefore, as memory improves so does judgment [17].

Comparison of the mean Cognistat scores here with those of known norms [18] reveals additional information about the effects of memory training. It is, for example, important to note whether improvement in memory here is great enough to remove individuals from MCI diagnosis. Interestingly, individuals do not improve to a memory "unimpaired" categorization. In fact, it is striking that the memory scores here are at least 2 standard deviations below the mean of neurologically intact older-adult populations while at the same time all other Cognistat subscale performance scores are approximately at the mean of the intact older-adult population. For example, the mean memory score for an older-adult sample is 10.1 with a 2.1 standard deviation [8], and a score of 9 or less indicating memory loss. Even though the present group improved their memory scores from 5.64 to 6.48, this did not approach the mean found within intact individuals [18]. That participants remain in the impaired range is not too surprising, however, given the initial trajectory of memory performance. Future work could train and test individuals over a longer time period in order to determine whether the memory improvement continues beyond 9 months [18]. Similarly, with regard to the subscales demonstrating decreasing performance as a function of time, individuals remained within the intact range, despite the decreasing scores. Thus, without memory 
training it would be predicted that the underlying likely progressive disease process would result in a global decrease in all cognitive domains; however, the present findings support that memory improves following memory training, while other cognitive domains continue their downward progression.

The biggest limitation of the current research is the absence of a control group. However, by comparing performance between cognitive domains (via the Cognistat subscales), we are able to examine any differential effects of memory training on cognitive domains. Such comparisons decrease the likelihood that any changes in task performance as a function of time reflect, for example, social interaction or some other extraneous factor, rather than the memory training itself. Given that memory improved relative to all other cognitive domains, with the exception of judgment, it is unlikely that factors other than memory training resulted in the findings reported here. We do acknowledge that this is a possibility however; perhaps the demand characteristics of the memory training procedure induced changes in memory, but not changes in other cognitive domains, though given the reality of cognitive decline, we find it unlikely that social demands or interaction could be enough to improve age-related cognitive impairment in memory, while other cognitive domains remain in decline. Nevertheless, we recommend that future studies include an explicit control group for the evaluation of the effects of memory training on cognition.

Furthermore, comparison of the present results here with those of other research supports our contention that it is memory training per se that resulted in the increased memory found here. For example, the results were consistent with the ACTIVE results, in which intact older adults were cognitively trained for 60 to 75 minute sessions over 5 to 6 week periods. Training was found to result in positive effects for up to 5 years [2] [4] [5]. Importantly, here MCI participants were given training for only 9 months, and results indicated similar findings in regard to memory improvement. Thus, in both the intact and MCI older-adult population, cognitive raining is beneficial. It is not clear yet whether memory training or some other forms of cognitive training would offer the most benefit to individuals with MCI. Regardless, it appears that cognitive training enhances performance on the cognitive domain undergoing training, and thus demonstrates specificity, rather than generalization. Thus, if a therapeutic goal is to change memory function, memory training is the treatment of choice, while if the goal is to modify another cognitive process, such as decision making, a different training procedure should be used. Future work could examine whether the type of memory that is improved can be changed based on the training procedure used, for example, whether semantic versus episodic memory can be differentially improved.

\section{Conclusion}

Memory training in older adult, MCI individuals improves memory, but does not improve other forms of cognition. Thus, cognitive training demonstrates specificity, rather than generalization, to the cognitive domain being trained. Results suggest that i) improvement of cognitive function in individuals with MCI is possible; ii) such improvement can be achieved in a relatively short period of time (e.g. 6 months); and iii) increased cognition in one domain may be unrelated to improvement in another cognitive domain. These results have important implications for the treatment of individuals with MCI.

\section{Acknowledgements}

The authors would like to thank Dr. Ruth E. Propper for statistical advising and assistance with manuscript preparation.

\section{References}

[1] Wolinsky, F.D., Vander Weg, M.W., Martin, R., Unverzagt, F.W., Willis, S.L., Marsiske, M., Tennstedt, S.L., et al. (2010) Does Cognitive Training Improve Internal Locus of Control Among Older Adults? The Journals of Gerontology Series B: Psychological Sciences and Social Sciences, 65B, 591-598. http://dx.doi.org/10.1093/geronb/gbp117

[2] Wolinsky, F.D., Unverzagt, F.W., Smith, D.M., Jones, R., Stoddard, A. and Tennstedt, S.L. (2006) The ACTIVE Cognitive Training Trial and Health-Related Quality of Life: Protection that Lasts for 5 Years. The Journals of Gerontology Series A: Biological Sciences and Medical Sciences, 61, 1324-1329. http://dx.doi.org/10.1093/gerona/61.12.1324

[3] Rebok, G.W., Ball, K., Guey, L.T., Jones, R.N., Kim, H.-Y., King, J.W., Willis, S.L., et al. (2014) Ten-Year Effects of the Advanced Cognitive Training for Independent and Vital Elderly Cognitive Training Trial on Cognition and Everyday Functioning in Older Adults. Journal of the American Geriatrics Society, 62, 16-24. http://dx.doi.org/10.1111/jgs.12607

[4] Ball, K., Berch, D.B., Helmers, K.F., Jobe, J.B., Leveck, M.D., Marsiske, M., et al. (2002) Effects of Cognitive Train- 
ing Interventions with Older Adults: A Randomized Controlled Trial. JAMA: The Journal of the American Medical Association, 288, 2271-2281. http://dx.doi.org/10.1001/jama.288.18.2271

[5] Willis, S.L., Tennstedt, S.L., Marsiske, M., Ball, K., Elias, J., Koepke, K.M., et al. (2006) Long-Term Effects of Cognitive Training on Everyday Functional Outcomes in Older Adults. JAMA: The Journal of the American Medical Association, 296, 2805-2814. http://dx.doi.org/10.1001/jama.296.23.2805

[6] Smith, G., Housen, P., Yaffe, K., Ruff, R., Kennison, R.F., Mahncke, H.W. and Zelinski, E.M. (2009) A Cognitive Training Program Based on Principles of Brain Plasticity: Results from the Improvement in Memory with Plasticity-Based Adaptive Cognitive Training (IMPACT) study. Journal of the American Geriatrics Society, 57, 594-603. http://dx.doi.org/10.1111/j.1532-5415.2008.02167.x

[7] Brehmer, Y., Rieckmann, A., Bellander, M., Westerberg, H., Fischer, H. and Bäckman, L. (2011) Neural Correlates of Training-Related Working-Memory Gains in Old Age. NeuroImage, 58, 1110-1120. http://dx.doi.org/10.1016/j.neuroimage.2011.06.079

[8] Richmond, L.L., Morrison, A.B., Chein, J.M. and Olson, I.R. (2011) Working Memory Training and Transfer in Older Adults. Psychology and Aging, 26, 813-822. http://dx.doi.org/10.1037/a0023631

[9] Magaro, P.A., Brotter, B. and Jalees, M. (2015) Memory Training and Task Specificity in 90-99-Year-Old Seniors with Mild Cognitive Impairment. Advances in Aging Research, 4, 1-12. http://dx.doi.org/10.4236/aar.2015.41001

[10] Kita, Y., Dodge, H.H., Ganguli, M., Takechi, H., Ueshima, H. and Hayakawa, T. (2008) Healthy Cognitive Aging and Leisure Activities among the Oldest Old in Japan: Takashima Study. The Journals of Gerontology Series A: Biological Sciences and Medical Sciences, 63, 1193-1200. http://dx.doi.org/10.1093/gerona/63.11.1193

[11] Yang, L. and Krampe, R.T. (2009) Long-Term Maintenance of Retest Learning in Young Old and Oldest Old Adults. The Journals of Gerontology Series B: Psychological Sciences and Social Sciences, 64B, 608-611. http://dx.doi.org/10.1093/geronb/gbp063

[12] Troyer, A.K., Murphy, K.J., Anderson, N.D., Moscovitch, M. and Craik, F.I.M. (2008) Changing Everyday Memory Behaviour in Amnestic Mild Cognitive Impairment: A Randomised Controlled Trial. Neuropsychological Rehabilitation, 18, 65-88. http://dx.doi.org/10.1080/09602010701409684

[13] Corrada, M.M., Berlau, D.J. and Kawas, C.H. (2012) A Population-Based Clinicopathological Study in the Oldest-Old: The 90+ Study. Current Alzheimer Research, 9, 709-717. http://dx.doi.org/10.2174/156720512801322537

[14] Prohovnik, I., Perl, D.P., Davis, K.L., Libow, L., Lesser, G. and Haroutunian, V. (2006) Dissociation of Neuropathology from Severity of Dementia in Late-Onset Alzheimer Disease. Neurology, 66, 49-55. http://dx.doi.org/10.1212/01.wnl.0000191298.68045.50

[15] Savva, G.M., Wharton, S.B., Ince, P.G., Forster, G., Matthews, F.E. and Brayne, C. (2009) Age, Neuropathology, and Dementia. The New England Journal of Medicine, 360, 2302-2309. http://dx.doi.org/10.1056/NEJMoa0806142

[16] Kwok, T.C.Y., Chau, W.W., Yuen, K.S.L., Wong, A.Y.M., Li, J.C.Y., Shiu, R.Y.Y. and Ho, F.K.Y. (2011) Who Would Benefit from Memory Training? A Pilot Study Examining the Ceiling Effect of Concurrent Cognitive Stimulation. Clinical Interventions in Aging, 6, 83-88.

[17] Moser, D.V. (1992) Does Memory Affect Judgment? Self-Generated versus Recall Memory Measures. Journal of Personality and Social Psychology, 62, 555-563. http://dx.doi.org/10.1037/0022-3514.62.4.555

[18] Kiernan, R.J., Mueller, J., Langston, J.W. and Van Dyke, C. (1997) The Neurobehavioral Cognitive Status Examination: A Brief But Differentiated Approach to Cognitive Assessment. Annals of Internal Medicine, 107, 481-485. http://dx.doi.org/10.7326/0003-4819-107-4-481 